\title{
Service Identification in SMEs: Appropriate Elements and Methods
}

\author{
Ali Taei Zadeh, Shahnorbanun Sahranb, and Muriati Mukhtar
}

\begin{abstract}
Implementing SOA in big enterprises are subjected to serious challenges such as a lack of comprehensive service governance road map and appropriate service identification method however the overall number of successes in service oriented solutions is increasing. Small medium enterprises (SME) on the other hand have specific challenges and problems that is peculiar to their nature. They have encountered serious problems in service modeling. Lack of researches in Service Identification is one of the first problem that an SME encounters when starting a SOA migration. Although a number of service identification methods have been proposed but they did not consider the SME challenges in those methods. Also the majority of those methods only considered the management point of view without considering a deep technical view. To fill this gap, this paper review existing service identification methods and proposes an improved method for identification of services based on SMEs specification and challenges.
\end{abstract}

Index Terms-Service identification method, SME, SOA, criteria.

\section{INTRODUCTION}

Service-oriented architecture (SOA) is an architectural approach to fill the gap between IT and business domain. The main SOA enhancements are agility in process and building compatible information systems with business changing frequency. This makes the enterprise has enough power to be competitive [1], [2]. SME has many influences to the world economy in both quantity and quality aspects. SMEs provide some SOA implementation requirements as nature properties. A study of 134 research paper mentioned that majority of SMEs have simple systems and processes, which allows flexibility, immediate feedback, short decision-making chain, better understanding and quicker response to customer needs than larger organizations [3]. These types of properties accelerate the SOA adaptation in SMEs. The most important factor in SOA success implementation is service modeling life cycle. That determines the services will have role in organization information system. Service identification method should be compatible with organization nature. The set of criteria of the method determines the size of service that effect on reusability and cohesion intensity [2].

SOA governance frameworks define all tasks and activities in a service oriented solutions such as design, control mechanism, SOA metrics, and security rules [4]. Many of

Manuscript received November 14, 2012; revised April 18, 2013.

A. T. Zadeh is with the UKM university, FTSM, Bangi 43000, Malaysia (e-mail: alitaee@gmail.com).

S. Sahranb and M. Mukhtar are with the Department of Industrial Computing, UKM university, FTSM, Bangi 43000, Malaysia (e-mail:shah@ftsm.ukm.my,mm@ftsm.ukm.my).
SOA governance frame works consider design services as first phase. So, other layers of SOA governance frameworks taking effect from the service identification results in service modeling stage. Many service Identification approaches has been proposed and each of those discuss selecting and extracting the services in a special point of view with completely difference in criteria and method that is used by them. On the other hand many identification approaches are based on business view and ignore technical view. As result translation from their output to enterprise executable environment is always ambiguous.

Also in order to satisfy small medium enterprises the identification approach should obviously consider economic aspects that it is ignored in some methods and mentioned in others but without considering SMEs specifications. Consequently, in order to achieve a suitable identification model for SMEs the following questions should be answered:

Q1: What kinds of services are suitable and appropriate to derived from an identification method for small medium enterprises?

Q2: what are the metrics should be considered in service identification method that is acceptable to business and technical domain?

The goal of this paper is evaluating existing service identification methods based on SMEs requirements and specification. And extracting influence criteria in SMEs domain as based for SMEs specific service identification method. To answer these questions and achieve the objectives we done a literature review and prepared a framework to compare existing identification methods.

This paper is organized as follows. Section II presents a literature review with a comparison mechanism of existing service identification methods by using new set of criteria that proposed based on SMEs requirements. Criteria for service identification method based on SMEs are illustrated in Section III. Accordingly the properties of new service identification method are presented in Section IV. Section V concludes the paper and looks at possible future works.

\section{PROCEDURe For PAPER SUBMISSION}

There are more than 50 works in service identification methods both in academic or industrial researches. And most of them are published in last four years that shows increasing attention to this area. Because of the nature of SOA that acts as bridge between IT and business, number of researches focuses on business view and others concentrate on technical view. Hubbers suggested ten common ways for identifying services [5]. The categorization is comprehensive and most of service identification methods use one of that ways. However 
some of them are not used by any service identification method [6]. Number of them that are more popular are based on business process view. Consequently, he focuses on business view that means top down approaches and as result ignored bottom up approaches and ignore to provide details about implementation. On the other hand the understanding of services is not same. Böhmann consider a service as independent object that done a process completely [7], [8]. But others believes that a service represent a special task with determined function whether it be a whole process or a part of it [9]. The scope of services is challenge in most of service identification methods and it is related to granularity of services [6]. So, granularity effects in usability and success of every service identification method. Also granularity has relation with reusability, flexibility, and complexity as shown in Fig. 1 [10].

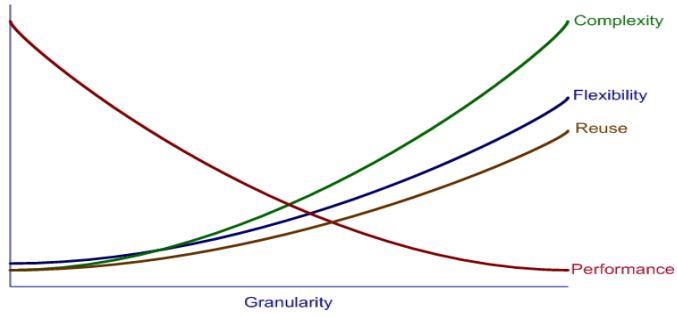

Fig. 1. Relationships between granularity and complexity, flexibility and reusability.

The right granularity and boundaries between services is not fixed. And it depends on the tolerant of complexity and intensity of tendency to reusability. On the other hand SMEs facing many challenges that could be covered by SOA.
Nach mentioned some challenges as lack in budget and expert people, absence of long term strategic planning and the defects in structure of SMEs [11]. However he neglect to present usable and practical instructions regarding to those difficulties.

The Lack of works in adopting SMEs with SOA leads high rate of failing in major SOA project in SMEs. Also shortcoming of researches on SOA in SMEs governance causes precaution in using SOA. However most of SME challenges could be addressed by applying SOA and reorganize their IT and business processes based on it Environmental uncertainties are one of SMEs challenges [11] That could be solved by implementing services in an integration environment.

\section{CRITERIA OF SERVICE IDENTIFICATION METHOD}

In the process of transition to service-oriented solution for SMEs we face set of challenges that should be considered in any solution. For example lack of experts, legacy soft ware's reusing, Interoperability, service boundaries, business and technical process supporting, economic Issues and etc. Table I categorizes these challenges in three set for better understanding. Some of these set of challenges are mentioned in related works and others are proposed as new in this paper with consideration SMEs environment [6]. There are completely differences in many subjects. Number of the important factors that is used by existing service identification is presented in Table I.

TABLE I: SERVICE IDENTIFICATION CHALLENGES AND THEIR REQUIREMENTS BASED ON SMES ENVIRONMENT

\begin{tabular}{|c|c|c|}
\hline Challenge & Challenge description & $\begin{array}{l}\text { Required } \\
\text { Capabilities }\end{array}$ \\
\hline \multicolumn{3}{|l|}{ General } \\
\hline Lack of experts & $\begin{array}{l}\text { Need workers with knowledge in both business and } \\
\text { technical aspects }\end{array}$ & Using Maximum automation approaches \\
\hline Service identification Road map & $\begin{array}{l}\text { Lack of clear strategies to implement service } \\
\text { identification method }\end{array}$ & $\begin{array}{l}\text { Present quantitative measurable } \\
\text { performance indicators instead of qualities } \\
\text { goals }\end{array}$ \\
\hline SOA delivery strategy & $\begin{array}{l}\text { Selection between top down, bottom up or mix } \\
\text { delivery strategy }\end{array}$ & $\begin{array}{l}\text { Identifying the business goals, process and } \\
\text { technical Issues }\end{array}$ \\
\hline \multicolumn{3}{|l|}{ Business } \\
\hline Business IT alignment & Gap between IT and business services & $\begin{array}{l}\text { Considering the bridge services between IT } \\
\text { and business services }\end{array}$ \\
\hline $\begin{array}{l}\text { Economic Issues } \\
\text { (Implement and maintenance costs) }\end{array}$ & Lack of budget in SMEs and strong competence & $\begin{array}{l}\text { Extract services with value creation, } \\
\text { Decreasing inter dependencies } \\
\text { external sourcing or shared services }\end{array}$ \\
\hline Lack of long-term planning and strategy & $\begin{array}{l}\text { High Rate of process changing and difficulties in } \\
\text { maintenance and updating identified services }\end{array}$ & $\begin{array}{l}\text { Special solutions for maintenance and } \\
\text { updating methods }\end{array}$ \\
\hline \multicolumn{3}{|l|}{ Technical } \\
\hline Stability & $\begin{array}{l}\text { Economic and technical aspects lead to less of } \\
\text { stability }\end{array}$ & $\begin{array}{l}\text { Providing stable infrastructure with } \\
\text { considering economic challenges }\end{array}$ \\
\hline Specifying services & Difficulties in determining specification of services & $\begin{array}{lll}\text { dynamically } & \text { discovering } & \text { service } \\
\text { specifications } & & \end{array}$ \\
\hline Complexity & $\begin{array}{l}\text { increasing in number of services in against limit } \\
\text { software }\end{array}$ & $\begin{array}{l}\text { Fine grained services } \\
\text { recognize inter dependent relations }\end{array}$ \\
\hline Service boundaries & $\begin{array}{l}\text { increase of complexity } \\
\text { decrease of reusability } \\
\text { decrease of flexibility } \\
\end{array}$ & Fine grained services based on the situation \\
\hline Interoperability & Heterogeneous hardware and software & Using General protocols \\
\hline
\end{tabular}


In addition, finding the links between inter-dependent challenges such as granularity and complexity and etc. will correct the direction of research efforts and it will be more precise.

The Lack of experts effects on all SOA migration steps. The nature of service-solutions is tied with need to IT and business experts. In addition for resuming the SOA governance in an organization, experts involving are required. On the other hand SMEs conditions do not allow expert participation in all steps of SOA migration.

So the maximum steps should be automate and experts involving needs should be decreased to as minimum as possible. Such characteristics confirm the identified services, service inter dependent, improve or adjust business process.

The Service boundaries depends to some subjects such as gaining maximum reusability that is important for SMEs in decreasing both technical and economic difficulties. But generally it relate to service definition, legal software, degree of inter connections between processes and etc. [12].

The service identification road map is ignored in most of existing service identification approaches. It helps to make a clear view for SOA identification phases and reduce the risks of implementation.

The SOA delivery strategy is discussed by many authors and it consist a fix place in any governance framework. Most of researches prefer top down delivery strategy to leverage business aspect against IT aspect that represent by bottom up approach [13]. Lack of long-term planning and strategy in SMEs is one of their specifications. It has bad effect on maintenance quality. This property increases the rate change of the identified process in SMEs. So more than big enterprises, SMEs need to solutions for maintenance and update for updating identified process. The delivery strategy is considered in all existing approaches. There are two major view points, business view and technical view.

In some approaches the attention is to answer business challenges and consider the goals of enterprise as highest priority. So top down approach are selected. On the contrary when using and integrating to legal software are more important the bottom up approach is used. In addition there are methods that uses mix delivery approach.

The Business IT alignment is major challenge before SOA solutions and remain after that based on research reports. However methods that based on business try to fill the business IT gap by give bigger role to business. The IT based methods believes versus. It seems that a comprehensive view could help to address this old challenge.

The Economic issues are very important factor and could be considered most important challenge in SMEs. Obviously majority of SMEs' specific challenges refers to budget problem. Due to long time and cyclic nature of service identification, it needs enough budgets that SMEs cannot afford. Consequently, SMEs need for tools and approaches that make this process shorter.

The interoperability is needed to support a heterogeneous environment in SMEs that often do not use ERP systems.

The stability becomes as challenge especially when the SMEs task are related to third parties. The weak infrastructure of SMEs force providing a solution for creates stable services.

The specifying services are a common phase in all service modeling methods. That determines the attributes of every service such as service ownership, service collaboration and etc. The requirements of this phase are difficult to gather from SMEs. For example good service abstraction is depending to the high level business requirements and goals that these are rarely found in SMEs before SOA implementation.

The complexity is related to service identification nature and some other challenges. Implementing and updating the identified process that is specially considered for SMEs increase the complexity. Editorial Policy

The submitting author is responsible for obtaining agreement of all coauthors and any consent required from sponsors before submitting a paper. It is the obligation of the authors to cite relevant prior work.

Authors of rejected papers may revise and resubmit them to the journal again.

\section{PROPOSED ELEMENTS FOR SERVICE IDENTIFICATION METHOD FOR SMES}

Researcher's tries to improve service identification methods based on challenges mentioned above. So researches propose number of methods. Most of frameworks that are proposed for service identification and mentioned in above sections focus on big enterprises requirements. Because there is no same point of view about service identification methods, these methods are different. These methods have been compared and the results have been presented in this section. The methods are called by the proposer name and those are proposed by Klose, Kohlmann, Amsden, Baghdadi, Jamshidi and Chen.

A special framework that is suitable for SMEs and cover their requirements could encourage them to cooperate in service solutions.

We propose appreciate elements extracted from existing service identification methods and new elements based on SME challenges that discussed in previous section. So a framework for evaluate the framework in order to identify appreciate elements are presented in Table II.

While the existing approaches discuss the general challenges but they did not mention the SMEs need. Only Kohlmann consider the IT business alignment by definition of both IT services and business process services. Kolmann with integration purpose tries to fill the gap between IT and business.

Economic issues within SOA implementation and maintenance did not considered in most approaches. Klose consider legacy systems and existing software applications that are suitable for SMEs as input because they have lack in their domain knowledge such as business models, requirements and etc.

Economic Issues, lack of experts and complexity have strong relationships that effect on each other directly. So any change in each of them has specific influence on others especially in SMEs. 
TABLE II: COMPARISON OF SERVICE IDENTIFICATION METHODS BASED ON SMES CHALLENGES

\begin{tabular}{|c|c|c|c|c|c|c|}
\hline \begin{tabular}{|ll} 
Challenges & Author \\
\end{tabular} & Klose & Kohlmann & Amsden & Jamshidi & Chen & Baghdadi \\
\hline Lack of experts & $\begin{array}{l}\text { Not } \\
\text { considered }\end{array}$ & Not considered & Not considered & \begin{tabular}{|l|} 
Automate \\
portion of \\
analysis steps
\end{tabular} & Not considered & Not considered \\
\hline Service identification Road map & analysis & guidelines & \begin{tabular}{|l|} 
Analysis \\
without precise \\
description
\end{tabular} & Algorithm & $\begin{array}{l}\text { Guidelines/ focus } \\
\text { on company's goals }\end{array}$ & $\begin{array}{l}\text { Present pattern without } \\
\text { validation }\end{array}$ \\
\hline SOA delivery strategy & Mix & Mix & Top down & Top down & Top down & Bottom up \\
\hline Business IT alignment & $\begin{array}{l}\text { Not } \\
\text { considered }\end{array}$ & $\left|\begin{array}{lr}\text { Define } & \text { both } \\
\text { and } & \text { business } \\
\text { process } & \text { services }\end{array}\right|$ & Not considered & Not considered & $\begin{array}{ll}\text { Based on } & \text { relations } \\
\text { power } & \text { between } \\
\text { services } & \end{array}$ & Not considered \\
\hline $\begin{array}{l}\text { Economic Issues } \\
\text { (Implement and maintenance costs) }\end{array}$ & $\begin{array}{|lr|}\text { Using } & \text { legacy } \\
\text { systems } & \text { as } \\
\text { input } & \\
\end{array}$ & Not considered & Not considered & Not considered & Not considered & Not considered \\
\hline Lack of long-term planning & $\begin{array}{ll}\text { Lines } & \text { of } \\
\text { interaction } & \& \\
\text { line } & \text { of } \\
\text { visibility } & \end{array}$ & $\begin{array}{l}\text { Sourcing } \\
\text { strategies }\end{array}$ & Not considered & Not considered & Not considered & Not considered \\
\hline Stability & $\begin{array}{l}\text { Not } \\
\text { considered }\end{array}$ & Not considered & Not considered & Not considered & Not considered & Not considered \\
\hline Specifying services & $\begin{array}{l}\text { Not } \\
\text { considered }\end{array}$ & Not considered & Not considered & \begin{tabular}{|l|} 
Limited details \\
about \\
dependencies
\end{tabular} & CRUD & $\begin{array}{l}\text { Determine their } \\
\text { operation and messages }\end{array}$ \\
\hline Complexity & $\begin{array}{l}\text { Not } \\
\text { considered }\end{array}$ & Not considered & Not considered & \begin{tabular}{|l} 
Algorithm \\
ambiguousness
\end{tabular} & Not considered & $\begin{array}{l}\text { Ambiguous } \\
\text { implementation details }\end{array}$ \\
\hline Service boundary & Middle & $\begin{array}{l}\text { Variablefrom } \\
\text { maximum to } \\
\text { middle }\end{array}$ & Not considered & $\begin{array}{ll}\text { Based } & \text { on } \\
\text { relations } & \\
\text { between } & \\
\text { services } & \end{array}$ & Not considered & Not considered \\
\hline Interoperability & $\begin{array}{l}\text { Not } \\
\text { considered }\end{array}$ & Not considered & Not considered & Not considered & Not considered & Not considered \\
\hline
\end{tabular}

Also service boundaries by determining size of services have relation with complexity. Maintenance the high number of services is difficult on the opposite side coarse grain services do not support the SOA benefits.

As result, the comparison shows necessity for service identification method that is compatible with SMEs condition and their requirements. That method should specify necessary elements that cover SOA-SMEs combination. So we addressed the main challenges of SMEs in SOA implementation in this paper. In addition suitable elements of existing service identification methods are extracted.

\section{CONCLUSION}

Service Oriented Architecture are used by many big enterprises in order to adopt with new business requirements On the other hand SMEs which formed a big sector of the business society have not really involved themselves in service solutions. Service Identification is a first step in entering service solutions and is important for SOA success and achieving its benefits. A number of Service Identification methods are proposed by the literature however these methods present SMEs with several challenges.

In this paper important challenges for service identification in SMEs are presented. In order to collect the suitable elements in existing methods, an analysis of compatibility level of these methods with SMEs challenges is shown. These elements could be considered in the first step to design method for SMEs.

\section{REFERENCES}

[1] N. Bieberstein, S. Bose, M. Fiammante, K. Jones, and R. Shah, Service-Oriented Architecture Compass: Business Value, Planning, and Enterprise Roadmap, Prentice-Hall, 2005.

[2] T. Erl, Service-Oriented Architecture: concepts, technology and design, NJ: Prentice Hall, Upper Saddle River, 2005.

[3] S. K. G. Rajesh, K. Singh, and S. G. Deshmukh, "Strategy development by SMEs for competitiveness: a review," Benchmarking: An International Journal, vol. 15, 2008.

[4] M. Niemann, "Governance for service-oriented architectures: an implementation approach," I-ESA2008, 2008.

[5] J. W. Hubbers et al., "Ten ways to identify services," SOA Magazine (XIII), 2007.

[6] Q. Gu and P. Lago, "Service identification methods: a systematic literature review," in Towards a Service-Based Internet, E. D. Nitto and R. Yahyapour, Eds. Springer Berlin / Heidelberg, 2010, vol. 6481, pp. 37-50.

[7] T. Herrmann et al., Konzepte für das Service Engineering: Modularisierung, Prozessgestaltung und Produktivitätsmanagement, Physica-Verlag Heidelberg, 2005.

[8] L. Bellissard et al., An Agent Platform for Reliable Asynchronous Distributed Programming, 1999, pp. 294-295.

[9] A. Arsanjani et al., "SOMA: A method for developing service-oriented solutions," IBM Systems Journal, vol. 47, pp. 377-396, 2008.

[10] R. Boerner and M. Goeken, "Service identification in SOA governance literature review and implications for a new method," in Proc. 2009, 
DEST '09. 3rd IEEE International Conf. on Digital Ecosystems and Technologies, 2009, pp. 588-593.

[11] H. Nach and A. Lejeune, "Implementing ERP in SMEs: Towards an Ontology Supporting Managerial Decisions," in Proc. 2008 International MCETECH Conf. on e-Technologies, 2008, pp. 223-226.

[12] P. Jamshidi et al., "To establish enterprise service model from enterprise business model," in Proc. 2008. SCC '08. IEEE International Conf. on Services Computing, 2008, pp. 93-100.

[13] J. Terlouw et al., An Assessment Method for Selecting an SOA Delivery Strategy: Determining Influencing Factors and Their Value Weights, 2009.

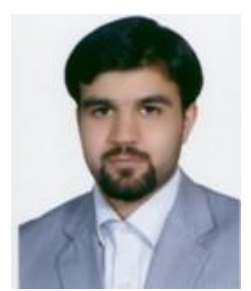

Ali Taei Zadeh is a Ph.D. candidate at the Department of Industrial Computing, National University of Malaysia (UKM). He received a master degree from the Islamic Azad University, Iran, in the field of System Engineering in 2008. His research interests include service modelind and software engineering. He works as a lecturer in Islamic Azad University in Iran. He is also a consultant in many projects as national or pilot in Iran. He published a number of papers in SOA modelind since 2009. 